\title{
On the mean value of the index of composition of an integer
}

by

Wenguang Zhai (Jinan)

1. Introduction. For each integer $n \geq 2$, let

$$
\lambda(n):=\frac{\log n}{\log \gamma(n)}
$$

be the index of composition of $n$, where $\gamma(n):=\prod_{p \mid n} p$. We write $\lambda(1)=$ $\gamma(1)=1$. The index of composition of an integer measures the multiplicity of its prime factors. De Koninck and Doyon [1] first studied the mean value of $\lambda(n)$. They proved the asymptotic formulas

$$
\sum_{n \leq x} \lambda(n)=x+c \frac{x}{\log x}+O\left(\frac{x}{\log ^{2} x}\right)
$$

and

$$
\sum_{n \leq x} \lambda^{-1}(n)=x+O\left(\frac{x}{\log x}\right),
$$

where $c=\sum_{p} \frac{\log p}{p(p-1)} \approx 0.75536$. These two asymptotic formulas imply that the average order of $\lambda(n)$ is 1 .

In [2] De Koninck and Kátai proved a series of results about the mean value of $\lambda(n)$. They proved that the asymptotic formulas

$$
\sum_{x<n \leq x+y} \lambda(n)=y+O\left(\frac{y}{\log x}\right)
$$

and

$$
\sum_{x<n \leq x+y} \lambda^{-1}(n)=y+O\left(\frac{y}{\log x}\right)
$$

hold for $y=x^{1 / 5} \log ^{3} x$. When $y=\sqrt{x}$, they proved that for any fixed

2000 Mathematics Subject Classification: Primary 11N37.

This work is supported by National Natural Science Foundation of China (Grant No. 10301018). 
integer $r \geq 1$, there exist computable constants $c_{1}, \ldots, c_{r}, d_{1}, \ldots, d_{r}$ such that

$$
\sum_{x<n \leq x+\sqrt{x}} \lambda(n)=\sqrt{x}+\sum_{j=1}^{r} c_{j} \frac{\sqrt{x}}{\log ^{j} x}+O\left(\frac{\sqrt{x}}{\log ^{r+1} x}\right)
$$

and

$$
\sum_{x<n \leq x+\sqrt{x}} \lambda^{-1}(n)=\sqrt{x}+\sum_{j=1}^{r} d_{j} \frac{\sqrt{x}}{\log ^{j} x}+O\left(\frac{\sqrt{x}}{\log ^{r+1} x}\right) .
$$

Then they deduced that:

$$
\sum_{n \leq x} \lambda(n)=x+\sum_{j=1}^{r} c_{j}^{\prime} \frac{x}{\log ^{j} x}+O\left(\frac{x}{\log ^{r+1} x}\right)
$$

and

$$
\sum_{n \leq x} \lambda^{-1}(n)=x+\sum_{j=1}^{r} d_{j}^{\prime} \frac{x}{\log ^{j} x}+O\left(\frac{x}{\log ^{r+1} x}\right)
$$

from $(1.5)$ and (1.6) respectively, where $c_{j}^{\prime}, d_{j}^{\prime}(j \geq 1)$ are computable constants.

In this paper we shall substantially improve the results of De Koninck and Kátai via a different approach, which is a variant of the Selberg method (see Ivić [4, Chapter 14]). By this approach we can also study the higher moments of $\lambda(n)$.

Before stating our main results, we introduce some notations. First we introduce the function

$$
H(u):=\prod_{p}\left(1-\frac{1}{p}\right)\left(1+\frac{1}{p-p^{u}}\right) \quad(-1<\Re u<1),
$$

which is very important in this paper. It is easily seen that $H(u)$ is regular in the range $-1<\Re u<1$ and

$$
H(0)=1, \quad H^{\prime}(0)=-\sum_{p} \frac{\log p}{p(p-1)} .
$$

Secondly, we introduce a series of integrals in order to study the positive moments of $\lambda(n)$. Suppose $f(u)$ is any function which is infinitely differentiable on $[-1 / 2,0], x \geq 2$. Define

$$
\begin{aligned}
& I_{1}(f, x):=\int_{-1 / 2}^{0} f(u) x^{u} d u, \\
& I_{2}(f, x):=\int_{-1 / 2}^{0} d u_{1} \int_{-1 / 2}^{u_{1}} f(u) x^{u} d u, \quad \ldots,
\end{aligned}
$$




$$
I_{k}(f, x):=\int_{-1 / 2}^{0} d u_{k-1} \int_{-1 / 2}^{u_{k-1}} \cdots \int_{-1 / 2}^{u_{2}} d u_{1} \int_{-1 / 2}^{u_{1}} f(u) x^{u} d u \quad(k \geq 2) .
$$

In Section 2 we shall prove the following

Proposition A. For any fixed integer $r \geq k$, we have

$$
I_{k}(f, x)=\sum_{l=k}^{r}\left(\begin{array}{l}
l-1 \\
k-1
\end{array}\right) \frac{(-1)^{l-k} f^{(l-k)}(0)}{\log ^{l} x}+O_{f, r}\left(\frac{1}{\log ^{r+1} x}\right) .
$$

Now we state our main results.

Theorem 1. Let $k \geq 1$ be a fixed integer. Then

$$
\sum_{n \leq x} \lambda^{k}(n)=\int_{2}^{x} I_{k}(H, z) \log ^{k} z d z+O\left(x^{1 / 2+\varepsilon}\right) .
$$

From Proposition A and Theorem 1 we immediately get

COROllary 1. Let $k, r \geq 1$ be fixed integers. Then

$$
\begin{aligned}
& \sum_{n \leq x} \lambda^{k}(n) \\
& \quad=x+\sum_{j=1}^{r}\left(\begin{array}{c}
j+k-1 \\
k-1
\end{array}\right)(-1)^{j} H^{(j)}(0) \int_{2}^{x} \frac{d z}{\log ^{j} z}+O\left(\frac{x}{\log ^{r+1} x}\right) .
\end{aligned}
$$

For the short interval case we have have

Theorem 2. Let $k \geq 1$ be a fixed integer. Then for $x^{1 / 5+\varepsilon} \leq y \leq x$, we

$$
\sum_{x<n \leq x+y} \lambda^{k}(n)=\int_{x}^{x+y} I_{k}(H, z) \log ^{k} z d z+O\left(y x^{-\varepsilon / 10}+x^{1 / 5+2 \varepsilon / 3}\right) .
$$

Corollary 2. Let $k, r \geq 1$ be fixed integers. Then for $x^{1 / 5+\varepsilon} \leq y \leq x$, we have

$$
\begin{aligned}
& \sum_{x<n \leq x+y} \lambda^{k}(n) \\
& =y+\sum_{j=1}^{r}\left(\begin{array}{c}
j+k-1 \\
k-1
\end{array}\right)(-1)^{j} H^{(j)}(0) \int_{x}^{x+y} \frac{d z}{\log ^{j} z}+O\left(\frac{y}{\log ^{r+1} x}\right) .
\end{aligned}
$$

Corollary 3. Let $k, r \geq 1$ be fixed integers. Then

$$
\sum_{n \leq x}\left(\lambda^{k}(n)-1\right)^{2}=\sum_{j=2}^{r} \alpha_{k, j}(-1)^{j} H^{(j)}(0) \int_{2}^{x} \frac{d z}{\log ^{j} z}+O\left(\frac{x}{\log ^{r+1} x}\right),
$$


where

$$
\alpha_{k, j}:=\left(\begin{array}{c}
j+2 k-1 \\
2 k-1
\end{array}\right)-2\left(\begin{array}{c}
j+k-1 \\
k-1
\end{array}\right) \quad(j \geq 2) .
$$

If $x^{1 / 5+\varepsilon} \leq y \leq x$, then

$$
\begin{aligned}
\sum_{x<n \leq x+y}\left(\lambda^{k}(n)\right. & -1)^{2} \\
& =\sum_{j=2}^{r} \alpha_{k, j}(-1)^{j} H^{(j)}(0) \int_{x}^{x+y} \frac{d z}{\log ^{j} z}+O\left(\frac{y}{\log ^{r+1} x}\right) .
\end{aligned}
$$

TheOREM 3. Let $k \geq 1$ be a fixed integer. Then

$$
\sum_{n \leq x} \lambda^{-k}(n)=x+\sum_{j=1}^{k}\left(\begin{array}{l}
k \\
j
\end{array}\right) H^{(j)}(0) \int_{2}^{x} \frac{d z}{\log ^{j} z}+O\left(x^{1 / 2+\varepsilon}\right) .
$$

TheOREM 4. Let $k \geq 1$ be a fixed integer. If $x^{1 / 5+\varepsilon} \leq y \leq x$, then

$$
\begin{aligned}
& \sum_{x<n \leq x+y} \lambda^{-k}(n) \\
& \quad=y+\sum_{j=1}^{k}\left(\begin{array}{l}
k \\
j
\end{array}\right) H^{(j)}(0) \int_{x}^{x+y} \frac{d z}{\log ^{j} z}+O\left(y x^{-\varepsilon / 10}+x^{1 / 5+2 \varepsilon / 3}\right) .
\end{aligned}
$$

From Theorems 3 and 4 we immediately get the following two corollaries.

Corollary 4. We have

$$
\sum_{n \leq x}\left(\lambda^{-1}(n)-1\right)^{2}=H^{(2)}(0) \int_{2}^{x} \frac{d z}{\log ^{2} z}+O\left(x^{1 / 2+\varepsilon}\right) .
$$

If $k \geq 2$, then

$$
\sum_{n \leq x}\left(\lambda^{-k}(n)-1\right)^{2}=\sum_{j=2}^{2 k} \beta_{k, j} H^{(j)}(0) \int_{2}^{x} \frac{d z}{\log ^{j} z}+O\left(x^{1 / 2+\varepsilon}\right),
$$

where

$$
\beta_{k, j}:= \begin{cases}\left(\begin{array}{c}
2 k \\
j
\end{array}\right)-2\left(\begin{array}{c}
k \\
j
\end{array}\right), & 2 \leq j \leq k \\
\left(\begin{array}{c}
2 k \\
j
\end{array}\right), & k+1 \leq j \leq 2 k .\end{cases}
$$

Corollary 5. Suppose $x^{1 / 5+\varepsilon} \leq y \leq x$. Then

$$
\sum_{x<n \leq x+y}\left(\lambda^{-1}(n)-1\right)^{2}=H^{(2)}(0) \int_{x}^{x+y} \frac{d z}{\log ^{2} z}+O\left(y x^{-\varepsilon / 10}+x^{1 / 5+2 \varepsilon / 3}\right)
$$


and for $k \geq 2$,

$$
\begin{aligned}
& \sum_{x<n \leq x+y}\left(\lambda^{-k}(n)-1\right)^{2} \\
& \quad=\sum_{j=2}^{2 k} \beta_{k, j} H^{(j)}(0) \int_{x}^{x+y} \frac{d z}{\log ^{j} z}+O\left(y x^{-\varepsilon / 10}+x^{1 / 5+2 \varepsilon / 3}\right) .
\end{aligned}
$$

REMARK 1. In [2], De Koninck and Kátai proved the long interval results (1.7) and (1.8) with the help of the short interval results (1.5) and (1.6), respectively. However, in this paper we shall use two different ways to study the long interval case and the short interval case.

REMARK 2. By repeated partial integration we get

$$
\int_{2}^{x} \frac{d z}{\log ^{j} z}=x \sum_{d=j}^{r} \frac{B_{d, j}}{\log ^{d} x}+O\left(\frac{x}{\log ^{r+1} x}\right)
$$

for any fixed $r \geq j$, where

$$
B_{d, j}:=(d-j) !\left(\begin{array}{l}
d-1 \\
d-j
\end{array}\right), \quad d \geq j .
$$

Our theorems and corollaries improve the results of De Koninck and Kátai. Furthermore, the constants $c_{j}, d_{j}, c_{j}^{\prime}, d_{j}^{\prime}(j \geq 1)$ in $(1.5)-(1.8)$ can be completely determined. Actually, taking $k=1$ in Corollaries 1 and 2 and Theorems 3 and 4 respectively and then using (1.21) we find easily that

$$
\begin{gathered}
c_{j}=(-1)^{j} H^{(j)}(0), \quad c_{j}^{\prime}=\sum_{i=1}^{j}(-1)^{i} H^{(i)}(0) B_{j, i} \quad(j \geq 1), \\
d_{1}=H^{\prime}(0), \quad d_{j}=0 \quad(j \geq 2), \quad d_{j}^{\prime}=H^{\prime}(0) B_{j, 1} \quad(j \geq 1) .
\end{gathered}
$$

Notations. Throughout this paper, $\varepsilon$ always denotes a fixed but sufficiently small positive constant; $\mu(n)$ is the Möbius function; $\mathrm{SC}\left(\sum\right)$ denotes the summation condition of the sum $\sum$. For any fixed integers $1 \leq a_{1} \leq$ $\cdots \leq a_{k}$

$$
d\left(a_{1}, \ldots, a_{k} ; n\right):=\sum_{n=n_{1}^{a_{1} \ldots n_{k}^{a_{k}}}} 1
$$

2. Preliminary results. The following two lemmas will be needed.

LEMma 1. Suppose $\sum_{n \leq x} f(n)=M(x)+O\left(x^{1 / 2+\varepsilon}\right)$, where $M \in C^{1}[1, \infty)$. Then for any fixed integer $\bar{j}$ we have

$$
\sum_{n \leq x} f(n) \log ^{j} n=\int_{2}^{x} M^{\prime}(z) \log ^{j} z d z+O\left(x^{1 / 2+\varepsilon}\right) .
$$

Proof. This follows easily from the Abelian summation formula. 
Lemma 2. Suppose $d \geq 2$ is a fixed integer and $x^{1 /(2 d+1)+\varepsilon} \leq y \leq x$. Then

$$
E(x, y, d ; \eta):=\sum_{\substack{x<n m^{d} \leq x+y \\ m>x^{\eta}}} 1 \ll y x^{-\eta}+x^{1 /(2 d+1)} \log x,
$$

where $\eta>0$ is a fixed small constant.

Proof. This is Lemma 3.3 of Zhai [5]. The exponent $1 /(2 d+1)$, which is connected with gap results for $d$-free numbers, is due to Filaseta and Trifonov [3].

Now we prove Proposition A. It is easy to show by partial integration that

$$
I_{k}(1, x)=\log ^{-k} x+O\left(x^{-1 / 2}\right)
$$

which implies that

$$
I_{k}(f, x) \ll_{f} \log ^{-k} x .
$$

We shall prove Proposition A by induction. By repeated partial summation we get

$$
I_{1}(f, x)=\sum_{j=1}^{r_{0}} \frac{(-1)^{j-1} f^{(j-1)}(0)}{\log ^{j} x}+O_{f, r_{0}}\left(\frac{1}{\log ^{r_{0}+1} x}\right)
$$

for any fixed $r_{0} \geq 1$. That is, the assertion of Proposition $\mathrm{A}$ is true for $k=1$.

Now suppose $k \geq 2$ and $r \geq k$ are fixed integers. By partial summation we get

$$
\int_{-1 / 2}^{u_{1}} f(u) x^{u} d u=\frac{x^{u_{1}} f\left(u_{1}\right)}{\log x}-\frac{1}{\log x} \int_{-1 / 2}^{u_{1}} f^{\prime}(u) x^{u} d u+O\left(x^{-1 / 2}\right) .
$$

Inserting this formula into the definition of $I_{k}(f, x)$ we get

$$
I_{k}(f, x)=\frac{I_{k-1}(f, x)}{\log x}-\frac{I_{k}\left(f^{\prime}, x\right)}{\log x}+O\left(x^{-1 / 2}\right) .
$$

Using this formula repeatedly we obtain

$$
\begin{aligned}
I_{k}(f, x)= & \sum_{j=1}^{r+1-k}(-1)^{j-1} \frac{I_{k-1}\left(f^{(j-1)}, x\right)}{\log ^{j} x}+(-1)^{r+1-k} \frac{I_{k}\left(f^{(r+1-k)}, x\right)}{\log ^{r+1-k} x} \\
& +O\left(x^{-1 / 2}\right) .
\end{aligned}
$$

Estimating the second term on the right-hand side by appealing to (2.2), we get

$$
I_{k}(f, x)=\sum_{j=1}^{r+1-k}(-1)^{j-1} \frac{I_{k-1}\left(f^{(j-1)}, x\right)}{\log ^{j} x}+O_{f, r}\left(\frac{1}{\log ^{r+1} x}\right) .
$$


If $k=2$, then (2.4) becomes

$$
I_{2}(f, x)=\sum_{j=1}^{r-1}(-1)^{j-1} \frac{I_{1}\left(f^{(j-1)}, x\right)}{\log ^{j} x}+O_{f, r}\left(\frac{1}{\log ^{r+1} x}\right) .
$$

Taking $r_{0}=r-j$ in $(2.3)$ we have

$$
I_{1}\left(f^{(j-1)}, x\right)=\sum_{i=1}^{r-j} \frac{(-1)^{i-1} f^{(j-1+i-1)}(0)}{\log ^{i} x}+O_{f, r}\left(\frac{1}{\log ^{r-j+1} x}\right) .
$$

From these two formulas we get

$$
\begin{aligned}
I_{2}(f, x) & =\sum_{\substack{i+j \leq r \\
i, j \geq 1}} \frac{(-1)^{i+j-2} f^{(i+j-2)}(0)}{\log ^{i+j} x}+O_{f, r}\left(\frac{1}{\log ^{r+1} x}\right) \\
& =\sum_{l=2}^{r}\left(\begin{array}{c}
l-1 \\
1
\end{array}\right) \frac{(-1)^{l-2} f^{(j-2)}(0)}{\log ^{l} x}+O_{f, r}\left(\frac{1}{\log ^{r+1} x}\right),
\end{aligned}
$$

that is, the case $k=2$ of Proposition $\mathrm{A}$ is true.

Now suppose the assertion is true for $k-1$. From this assumption we get

$$
\begin{aligned}
& I_{k-1}\left(f^{(j-1)}, x\right) \\
& \quad=\sum_{l_{1}=k-1}^{r-j}\left(\begin{array}{l}
l_{1}-1 \\
k-2
\end{array}\right) \frac{(-1)^{l_{1}-k+1} f^{\left(j-1+l_{1}-k+1\right)}(0)}{\log ^{l_{1}} x}+O_{f, r}\left(\frac{1}{\log ^{r-j+1} x}\right),
\end{aligned}
$$

which combined with (2.4) gives

$$
\begin{aligned}
& I_{k}(f, x) \\
& =\sum_{j=1}^{r+1-k} \sum_{l_{1}=k-1}^{r-j}\left(\begin{array}{c}
l_{1}-1 \\
k-2
\end{array}\right) \frac{(-1)^{j+l_{1}-k} f^{\left(j+l_{1}-k\right)}(0)}{\log ^{j+l_{1}} x}+O_{f, r}\left(\frac{1}{\log ^{r+1} x}\right) \\
& =\sum_{l=k}^{r} B(l, k) \frac{(-1)^{l-k} f^{(l-k)}(0)}{\log ^{l} x}+O_{f, r}\left(\frac{1}{\log ^{r+1} x}\right),
\end{aligned}
$$

where

$$
B(l, k)=\sum_{l_{1}=k-1}^{l-1}\left(\begin{array}{l}
l_{1}-1 \\
k-2
\end{array}\right) \quad(l \geq k) .
$$

Suppose $|t|<1$. Then

$$
\frac{t^{k}}{(1-t)^{k}}=\sum_{l=k}^{\infty}\left(\begin{array}{l}
l-1 \\
k-1
\end{array}\right) t^{l}
$$


Writing $\frac{t^{k}}{(1-t)^{k}}=\frac{t}{1-t} \cdot \frac{t^{k-1}}{(1-t)^{k-1}}$ we get

$$
\sum_{l=k}^{\infty}\left(\begin{array}{l}
l-1 \\
k-1
\end{array}\right) t^{l}=\sum_{j=1}^{\infty} t^{j} \cdot \sum_{l_{1}=k-1}^{\infty}\left(\begin{array}{l}
l_{1}-1 \\
k-2
\end{array}\right) t^{l_{1}}
$$

which implies $B(l, k)=\left(\begin{array}{l}l-1 \\ k-1\end{array}\right)$. Thus the assertion of Proposition $\mathrm{A}$ is true for $k$.

3. Mean value of $\gamma^{u}(n)$. Suppose $u \in \mathbb{C}$ with $-1<\Re u<1$. For each fixed $u, \gamma^{u}(n)$ is a multiplicative function of $n$. For each $n, \gamma^{u}(n)$ is a regular function of $u$.

We first prove

Proposition B. If $-1+\varepsilon<\Re u<1-\varepsilon$, then

$$
\sum_{n \leq x} \gamma^{u}(n)=H_{1}(u) x^{1+u}+O\left(x^{1 / 2+\max (\Re u, \Re u / 2)+\varepsilon}\right),
$$

where $H_{1}(u):=H(u) /(1+u)$ and the $O$-term is uniform in $u$.

Proof. Let $s$ and $u$ be complex numbers with $\Re(s-u)>1$, and define

$$
G(s, u):=\sum_{n=1}^{\infty} \frac{\gamma^{u}(n)}{n^{s}} .
$$

By Euler product representation we have

$$
G(s, u)=\prod_{p}\left(1+\frac{p^{u}}{p^{s}}+\frac{p^{u}}{p^{2 s}}+\frac{p^{u}}{p^{3 s}}+\cdots\right)=\zeta(s-u) G^{*}(s, u),
$$

say, where

$$
\begin{aligned}
G^{*}(s, u) & :=\prod_{p}\left(1-\frac{p^{u}}{p^{s}}\right)\left(1+\frac{p^{u}}{p^{s}}+\frac{p^{u}}{p^{2 s}}+\frac{p^{u}}{p^{3 s}}+\cdots\right) \\
& =\prod_{p}\left(1-\frac{p^{u}}{p^{s}}\right)\left(1+\frac{p^{u}}{p^{s}-1}\right) .
\end{aligned}
$$

By the definition of $H(u)$ we have

$$
G^{*}(1+u, u)=H(u) .
$$

It is easy to show that the infinite product on the right-hand side of (3.3) is absolutely convergent in the range $\Re s>1 / 2+\max (\Re u, \Re u / 2)$. Write

$$
G^{*}(s, u)=\sum_{n=1}^{\infty} \frac{b(n, u)}{n^{s}}
$$


this infinite series is also absolutely convergent in the same range. This implies that

$$
\sum_{n \leq x}|b(n, u)|=O\left(x^{1 / 2+\max (\Re u, \Re u / 2)+\varepsilon}\right) .
$$

Note that $\zeta(s-u)=\sum_{n=1}^{\infty} n^{u} / n^{s}$. For $-1<\Re u<1$, by the Euler-Maclaurin formula we easily get

$$
\sum_{n \leq x} n^{u}=\frac{x^{1+u}}{1+u}+O\left(\max \left(1, x^{\Re u}\right)\right) .
$$

From (3.2) and (3.4)-(3.6) we obtain

$$
\begin{aligned}
\sum_{n \leq x} \gamma^{u}(n) & =\sum_{m \leq x} b(m, u) \sum_{n \leq x / m} n^{u} \\
& =\sum_{m \leq x} b(m, u)\left(\frac{1}{1+u} \frac{x^{1+u}}{m^{1+u}}+O\left(\max \left(1, \frac{x^{\Re u}}{m^{\Re u}}\right)\right)\right) \\
& =\frac{x^{1+u}}{1+u} \sum_{m \leq x} \frac{b(m, u)}{m^{1+u}}+O\left(x^{1 / 2+\max (\Re u, \Re u / 2)+\varepsilon}\right) \\
& =H_{1}(u) x^{1+u}+O\left(x^{1 / 2+\max (\Re u, \Re u / 2)+\varepsilon}\right)
\end{aligned}
$$

if we note that

$$
\sum_{m \leq x} \frac{b(m, u)}{m^{1+u}}=G^{*}(1+u, u)+O\left(x^{-1 / 2+\max (\Re u, \Re u / 2)-\Re u+\varepsilon),}\right.
$$

which follows from (3.5) easily. This completes the proof of Proposition B.

Proposition C. If $-5 / 9 \leq \Re u \leq \varepsilon^{2}, x^{1 / 5+\varepsilon} \leq y \leq x$, then

$$
\sum_{x<n \leq x+y} \gamma^{u}(n)=H(u) \int_{x}^{x+y} z^{u} d z+O\left(y x^{-\varepsilon / 10}+x^{1 / 5+2 \varepsilon / 3}\right),
$$

where the $O$-term is uniform in $u$.

Proof. If $x^{3 / 4} \leq y \leq x$, then from Proposition B we have

$$
\begin{aligned}
\sum_{x<n \leq x+y} \gamma^{u}(n) & =H_{1}(u)(x+y)^{1+u}-H_{1}(u) x^{1+u}+O\left(x^{2 / 3}\right) \\
& =H(u) \int_{x}^{x+y} z^{u} d z+O\left(y x^{-\varepsilon / 2}\right) .
\end{aligned}
$$

Now suppose $x^{1 / 5+\varepsilon} \leq y \leq x^{5 / 6}$. It is easily seen that

$$
\sum_{x<n \leq x+y} \gamma^{u}(n)=\sum_{1} m^{u} \gamma^{u}(l)|\mu(m)|
$$

$\mathrm{SC}\left(\sum_{1}\right): x<m l \leq x+y,(m, l)=1, l$ is square-full, 
if we note that $\gamma(m)=m$ for square-free $m$. Since

$$
\sum_{l \text { square-full }} l^{-s}=\frac{\zeta(2 s) \zeta(3 s)}{\zeta(6 s)}
$$

we can write

$$
\begin{aligned}
\sum_{x<n \leq x+y} \gamma^{u}(n) & =\sum_{\substack{x<m n_{1}^{2} n_{2}^{3} n_{3}^{6} \leq x+y \\
\left(m, n_{1}^{2} n_{2}^{3} n_{3}^{6}\right)=1}} m^{u} \gamma^{u}\left(n_{1}^{2} n_{2}^{3} n_{3}^{6}\right)|\mu(m)| \mu\left(n_{3}\right) \\
& =\sum_{2}+O\left(x^{\varepsilon^{2}}\left(E_{1}+E_{2}+E_{3}\right)\right)
\end{aligned}
$$

where

$$
\begin{aligned}
& \sum_{2}:=\sum_{n_{1}, n_{2}, n_{3} \leq x^{\varepsilon / 2}} \gamma^{u}\left(n_{1}^{2} n_{2}^{3} n_{3}^{6}\right) \mu\left(n_{3}\right) \sum_{3} m^{u}|\mu(m)|, \\
& \mathrm{SC}\left(\sum_{3}\right): x\left(n_{1}^{2} n_{2}^{3} n_{3}^{6}\right)^{-1}<m \leq(x+y)\left(n_{1}^{2} n_{2}^{3} n_{3}^{6}\right)^{-1},\left(m, n_{1}^{2} n_{2}^{3} n_{3}^{6}\right)=1, \\
& E_{j}:=\sum_{\substack{x<m n_{1}^{2} n_{2}^{3} n_{3}^{6} \leq x+y \\
n_{j}>x^{\varepsilon / 2}}} 1 \quad(j=1,2,3) .
\end{aligned}
$$

By Lemma 2 we have

$$
\begin{aligned}
& E_{1}=\sum_{\substack{x<m n_{1}^{2} n_{2}^{3} n_{3}^{6} \leq x+y \\
n_{1}>x^{\varepsilon / 2}}} 1=\sum_{\substack{x<m n_{1}^{2} \leq x+y \\
n_{1}>x^{\varepsilon / 2}}} d(1,3,6 ; m) \\
& \ll x^{\varepsilon^{2}} E(x, y, 2 ; \varepsilon / 2) \ll y x^{-\varepsilon / 2+\varepsilon^{2}}+x^{1 / 5+\varepsilon^{2}} \log x
\end{aligned}
$$

if we note that $d(1,3,6 ; m) \ll m^{\varepsilon^{2}}$. Similarly

$$
\begin{aligned}
& E_{2} \ll x^{\varepsilon^{2}} E(x, y, 3 ; \varepsilon / 2) \ll y x^{-\varepsilon / 2+\varepsilon^{2}}+x^{1 / 7+\varepsilon^{2}} \log x, \\
& E_{3} \ll x^{\varepsilon^{2}} E(x, y, 6 ; \varepsilon / 2) \ll y x^{-\varepsilon / 2+\varepsilon^{2}}+x^{1 / 13+\varepsilon^{2}} \log x .
\end{aligned}
$$

Suppose $D \geq 1$ is an integer. For $\Re(s-u)>1$, consider

$$
G_{1}(s, u ; D):=\sum_{\substack{m=1 \\(m, D)=1}}^{\infty} \frac{m^{u}|\mu(m)|}{m^{s}} .
$$

By Euler product representation we have

$$
\begin{aligned}
G_{1}(s, u ; D) & =\prod_{\substack{(p, D)=1 \\
p^{s}}}\left(1+\frac{p^{u}}{p^{s}}\right) \prod_{\substack{(p, D)=1 \\
p^{s}}}\left(1-\frac{p^{u}}{p^{-1}} \prod_{(p, D)=1}\left(1-\frac{p^{2 u}}{p^{2 s}}\right)\right. \\
& =\sum_{\substack{n=1 \\
(n, D)=1}}^{\infty} \frac{n^{u}}{n^{s}} \cdot \sum_{\substack{l=1 \\
(l, D)=1}}^{\infty} \frac{\mu(l) l^{2 u}}{l^{2 s}} .
\end{aligned}
$$


Thus we may write

$$
\begin{gathered}
\sum_{\substack{x / D<m \leq(x+y) / D \\
(m, D)=1}} m^{u}|\mu(m)|=\sum_{\substack{x / D<n_{4} n_{5}^{2} \leq(x+y) / D \\
\left(n_{4}, D\right)=\left(n_{5}, D\right)=1}} n_{4}^{u} n_{5}^{2 u} \mu\left(n_{5}\right) \\
=\sum_{4}+O\left((x / D)^{\varepsilon^{2}} E_{4}\right), \\
\sum_{4}:=\sum_{\substack{n_{5} \leq(x / D)^{\varepsilon} \\
\left(n_{5}, D\right)=1}} n_{5}^{2 u} \mu\left(n_{5}\right) \sum_{\substack{x / D n_{5}^{2}<n_{4} \leq(x+y) / D n_{5}^{2} \\
\left(n_{4}, D\right)=1}} n_{4}^{u}, \\
E_{4}:=E(x / D, y / D, 2 ; \varepsilon) \ll y x^{-\varepsilon} D^{-1+\varepsilon}+x^{1 / 5} D^{-1 / 5} \log x .
\end{gathered}
$$

By the well known formula $\sum_{d \mid l} \mu(d)=[1 / l]$ and (3.6) we get

$$
\begin{aligned}
\sum_{\substack{n \leq T \\
(n, \bar{D})=1}} n^{u} & =\sum_{n \leq T} \sum_{d \mid(n, D)} \mu(d)=\sum_{d \mid D} \mu(d) d^{u} \sum_{n \leq T / d} n^{u} \\
& =\sum_{d \mid D} \mu(d) d^{u}\left(\frac{1}{1+u} \frac{T^{1+u}}{d^{1+u}}+O\left(\max \left(1, \frac{T^{\Re u}}{d^{\Re u}}\right)\right)\right) \\
& =\frac{\varrho_{1}(D) T^{1+u}}{1+u}+O\left(2^{\omega(D)} \max \left(1, T^{\Re u}\right)\right),
\end{aligned}
$$

where

$$
\varrho_{1}(D):=\sum_{d \mid D} \frac{\mu(d)}{d}
$$

Thus we get

$$
\begin{aligned}
& \sum_{\substack{x / D n_{5}^{2}<n_{4} \leq(x+y) / D n_{5}^{2} \\
\left(n_{4}, D\right)=1}} n_{4}^{u} \\
& =\frac{\varrho_{1}(D)}{D^{1+u} n_{5}^{2+2 u}} \int_{x}^{x+y} z^{u} d z+O\left(2^{\omega(D)} \max \left(1,\left(\frac{x}{D n_{5}^{2}}\right)^{\Re u}\right)\right) .
\end{aligned}
$$

Inserting (3.16) into the definition of $\sum_{4}$ we obtain

$$
\begin{aligned}
\sum_{4} & =\frac{\varrho_{1}(D)}{D^{1+u}} \sum_{\substack{n_{5} \leq(x / D)^{\varepsilon} \\
\left(n_{5}, D\right)=1}} \frac{\mu\left(n_{5}\right)}{n_{5}^{2}} \int_{x}^{x+y} z^{u} d z+O\left(2^{\omega(D)}(x / D)^{\varepsilon+\varepsilon^{2}}\right) \\
& =\frac{\varrho_{1}(D) \varrho_{2}(D)}{D^{1+u}} \int_{x}^{x+y} z^{u} d z+O\left(y x^{-\varepsilon+\varepsilon^{2}} D^{-1+\varepsilon-\varepsilon^{2}}\right)
\end{aligned}
$$


where

$$
\varrho_{2}(D):=\sum_{\substack{n_{5}=1 \\\left(n_{5}, D\right)=1}}^{\infty} \frac{\mu\left(n_{5}\right)}{n_{5}^{2}} .
$$

From (3.14) and (3.17) we get

$$
\begin{aligned}
\sum_{\substack{x<m \leq(x+y) / D \\
(m, D)=1}} & m^{u}|\mu(m)| \\
= & \frac{\varrho_{1}(D) \varrho_{2}(D)}{D^{1+u}} \int_{x}^{x+y} z^{u} d z+O\left(y x^{-\varepsilon+\varepsilon^{2}} D^{-1+\varepsilon-\varepsilon^{2}}\right) \\
& +O\left(x^{1 / 5+\varepsilon^{2}} D^{-1 / 5-\varepsilon^{2}} \log x\right),
\end{aligned}
$$

which combined with (3.10)-(3.13) gives

$$
\begin{aligned}
\sum_{x<n \leq x+y} \gamma^{u}(n)= & \sum_{5}+O\left(y x^{-\varepsilon+\varepsilon^{2}} \sum_{n_{1}, n_{2}, n_{3} \leq x^{\varepsilon / 2}}\left(n_{1}^{2} n_{2}^{3} n_{3}^{6}\right)^{-1+\varepsilon-\varepsilon^{2}}\right) \\
& +O\left(x^{1 / 5+\varepsilon^{2}} \sum_{n_{1}, n_{2}, n_{3} \leq x^{\varepsilon / 2}}\left(n_{1}^{2} n_{2}^{3} n_{3}^{6}\right)^{-1 / 5-\varepsilon^{2}} \log x\right) \\
= & \sum_{5}+O\left(y x^{-\varepsilon+\varepsilon^{2}}+x^{1 / 5+\varepsilon / 2+\varepsilon^{2}}\right) \\
= & \sum_{5}+O\left(y x^{-\varepsilon / 3}+x^{1 / 5+2 \varepsilon / 3}\right),
\end{aligned}
$$

where

$$
\sum_{5}=\sum_{n_{1}, n_{2}, n_{3} \leq x^{\varepsilon / 2}} \frac{\gamma^{u}\left(n_{1}^{2} n_{2}^{3} n_{3}^{6}\right) \mu\left(n_{3}\right) \varrho_{1}\left(n_{1}^{2} n_{2}^{3} n_{3}^{6}\right) \varrho_{2}\left(n_{1}^{2} n_{2}^{3} n_{3}^{6}\right)}{\left(n_{1}^{2} n_{2}^{3} n_{3}^{6}\right)^{1+u}} \int_{x}^{x+y} z^{u} d z .
$$

Suppose $-1<v \leq 1 / 2$. Consider

$$
G_{2}(s, v):=\sum_{n=1}^{\infty} \frac{\gamma^{v}(n) d(2,3,6 ; n)}{n^{s}}, \quad \Re(s-v)>1 .
$$

For $\alpha \geq 2$, let $N(\alpha)$ denote the number of non-negative solutions of the equation $2 l_{1}+3 l_{2}+6 l_{3}=\alpha$. By Euler product representation we have

$$
\begin{aligned}
G_{2}(s, v) & =\prod_{p}\left(1+\sum_{\alpha=2}^{\infty} \frac{p^{v} N(\alpha)}{p^{\alpha s}}\right) \\
& =\prod_{p}\left(1+\frac{p^{v}}{p^{2 s}}+\frac{p^{v}}{p^{3 s}}+\frac{p^{v}}{p^{4 s}}+\frac{p^{v}}{p^{5 s}}+\frac{3 p^{v}}{p^{6 s}}+\cdots\right) \\
& =\zeta(2 s-v) G_{3}(s, v),
\end{aligned}
$$


where

$$
G_{3}(s, v):=\prod_{p}\left(1-\frac{p^{v}}{p^{2 s}}\right)\left(1+\sum_{\alpha=2}^{\infty} \frac{p^{v} N(\alpha)}{p^{\alpha s}}\right) .
$$

It is easy to show that if we write $G_{3}(s, v)$ as a Dirichlet series, then this series is absolutely convergent for $\Re s>(1+v) / 3$. Thus by Perron's formula we easily get

$$
\begin{aligned}
\sum_{n \leq T} \gamma^{v}(n) d(2,3,6 & ; n) \\
& =\frac{G_{3}((1+v) / 2, v)}{1+v} T^{(1+v) / 2}+O\left(T^{(1+v) / 3+\varepsilon}\right),
\end{aligned}
$$

which implies that the infinite series

$$
\sum_{n_{1}, n_{2}, n_{3}} \frac{\gamma^{u}\left(n_{1}^{2} n_{2}^{3} n_{3}^{6}\right) \mu\left(n_{3}\right) \varrho_{1}\left(n_{1}^{2} n_{2}^{3} n_{3}^{6}\right) \varrho_{2}\left(n_{1}^{2} n_{2}^{3} n_{3}^{6}\right)}{\left(n_{1}^{2} n_{2}^{3} n_{3}^{6}\right)^{1+u}}
$$

is absolutely convergent if we note that $\varrho_{1}(n) \ll 1, \varrho_{2}(n) \ll 1$. Let $H^{*}(u)$ denote this infinite series. By (3.22) and partial summation we easily get

$$
\begin{aligned}
\left|\sum_{n_{1}, n_{2}, n_{3} \leq x^{\varepsilon / 2}} \frac{\gamma^{u}\left(n_{1}^{2} n_{2}^{3} n_{3}^{6}\right) \mu\left(n_{3}\right) \varrho_{1}\left(n_{1}^{2} n_{2}^{3} n_{3}^{6}\right) \varrho_{2}\left(n_{1}^{2} n_{2}^{3} n_{3}^{6}\right)}{\left(n_{1}^{2} n_{2}^{3} n_{3}^{6}\right)^{1+u}}-H^{*}(u)\right| \\
\leq \sum_{n>x^{\varepsilon / 2}} \frac{\gamma^{\Re u}(n) d(2,3,6 ; n)}{n^{1+\Re u}} \ll x^{-(1+\Re u) \varepsilon / 4} \ll x^{-\varepsilon / 9}
\end{aligned}
$$

if we note that $\Re u \geq-5 / 9$. From (3.19), (3.20) and (3.23) we get

$$
\sum_{x<n \leq x+y} \gamma^{u}(n)=H^{*}(u) \int_{x}^{x+y} z^{u} d z+O\left(y x^{-\varepsilon / 10}+x^{1 / 5+2 \varepsilon / 3}\right),
$$

and comparison with (3.9) gives $H^{*}(u)=H(u)$. This completes the proof of Proposition C.

4. Proofs of theorems. We first prove Theorem 1 . Let $\Delta(x, u)$ denote the error term in (3.1). By the definition of $I_{k}(f, x)$ we get

$$
\begin{aligned}
& \sum_{2 \leq n \leq x} I_{k}(1, \gamma(n))=M_{k}(x)+\Delta^{*}(x), \\
& M_{k}(x):=\int_{-1 / 2}^{0} d u_{k-1} \int_{-1 / 2}^{u_{k-1}} \cdots \int_{-1 / 2}^{u_{2}} d u_{1} \int_{-1 / 2}^{u_{1}} H_{1}(u) x^{1+u} d u, \\
& \Delta^{*}(x):=\int_{-1 / 2}^{0} d u_{k-1} \int_{-1 / 2}^{u_{k-1}} \cdots \int_{-1 / 2}^{u_{2}} d u_{1} \int_{-1 / 2}^{u_{1}} \Delta(x, u) d u \ll x^{1 / 2+\varepsilon} .
\end{aligned}
$$


By (2.1) and Proposition B with $u=-1 / 2$ the left-hand side of (4.1) is

$$
\sum_{2 \leq n \leq x}\left(\frac{1}{\log ^{k} \gamma(n)}+O\left(\gamma^{-1 / 2}(n)\right)\right)=\sum_{2 \leq n \leq x} \frac{1}{\log ^{k} \gamma(n)}+O\left(x^{1 / 2+\varepsilon}\right) .
$$

It is easily seen that $d M_{k}(x)=I_{k}(H, x) d x$. Thus Theorem 1 follows from the above formulas and Lemma 1. Similarly Theorem 2 follows from Proposition C.

Now we prove Theorem 3 . The function $H_{1}(u) x^{1+u}$ can be written as

$$
x \sum_{m=0}^{\infty} \frac{H_{1}^{(m)}(0)}{m !} u^{m} \sum_{n=0}^{\infty} \frac{\log ^{n} x}{n !} u^{n}=x \sum_{m, n}\left(\frac{H_{1}^{(m)}(0)}{m !} \frac{\log ^{n} x}{n !}\right) u^{m+n} .
$$

Taking the $k$ th derivative of both sides of (3.1) and then letting $u=0$ we get

$$
\begin{aligned}
\sum_{n \leq x} \log ^{k} \gamma(n) & =x \sum_{m+n=k} \frac{k ! H_{1}^{(m)}(0) \log ^{n} x}{m ! n !}+O\left(x^{1 / 2+\varepsilon}\right) \\
& =x \sum_{j=0}^{k}\left(\begin{array}{l}
k \\
j
\end{array}\right) H_{1}^{(j)}(0) \log ^{k-j} x+O\left(x^{1 / 2+\varepsilon}\right),
\end{aligned}
$$

which combined with Lemma 1 gives

$$
\begin{aligned}
& \sum_{n \leq x} \lambda^{-k}(n)=1+\sum_{2 \leq n \leq x} \frac{\log ^{k} \gamma(n)}{\log ^{k} n} \\
& =\int_{2}^{x} \frac{1}{\log ^{k} z} d \sum_{j=0}^{k}\left(\begin{array}{c}
k \\
j
\end{array}\right) H_{1}^{(j)}(0) z \log ^{k-j} z+O\left(x^{1 / 2+\varepsilon}\right) \\
& =\sum_{j=0}^{k}\left(\begin{array}{c}
k \\
j
\end{array}\right) H_{1}^{(j)}(0) \int_{2}^{x} \frac{\log ^{k-j} z}{\log ^{k} z} d z+O\left(x^{1 / 2+\varepsilon}\right) \\
& =\sum_{j=0}^{k}\left(\begin{array}{c}
k \\
j
\end{array}\right) H_{1}^{(j)}(0) \int_{2}^{x} \frac{\log ^{k-j} z+(k-j) \log ^{k-j-1} z}{\log ^{k} z} d z+O\left(x^{1 / 2+\varepsilon}\right) \\
& =\sum_{j=0}^{k}\left(\begin{array}{c}
k \\
j
\end{array}\right) H_{1}^{(j)}(0) \int_{2}^{x} \frac{d z}{\log ^{j} z}+\sum_{j=0}^{k}(k-j)\left(\begin{array}{c}
k \\
j
\end{array}\right) H_{1}^{(j)}(0) \int_{2}^{x} \frac{d z}{\log ^{j+1} z}+O\left(x^{1 / 2+\varepsilon}\right) \\
& =x+\sum_{j=1}^{k} c_{k, j} \int_{2}^{x} \frac{d z}{\log ^{j} z}+O\left(x^{1 / 2+\varepsilon}\right)
\end{aligned}
$$

where

$$
\begin{aligned}
c_{k, j} & =\left(\begin{array}{c}
k \\
j
\end{array}\right) H_{1}^{(j)}(0)+(k-j+1)\left(\begin{array}{c}
k \\
j-1
\end{array}\right) H_{1}^{(j-1)}(0) \\
& =\left(\begin{array}{c}
k \\
j
\end{array}\right) H_{1}^{(j)}(0)+j\left(\begin{array}{c}
k \\
j
\end{array}\right) H_{1}^{(j-1)}(0) .
\end{aligned}
$$


Note that $H(u)=H_{1}(u)(1+u)$. Applying Leibniz's formula to this equation and then taking $u=0$ we get $H^{(j)}(0)=H_{1}^{(j)}(0)+j H_{1}^{(j-1)}(0)$, which implies $c_{k, j}=\left(\begin{array}{c}k \\ j\end{array}\right) H^{(j)}(0)$. This completes the proof of Theorem 3 .

Finally, we prove Theorem 4. If $x^{1 / 2+\varepsilon} \leq y \leq x$, then the conclusion of Theorem 4 follows from Theorem 3 directly. Now suppose $x^{1 / 5+\varepsilon} \leq y \leq$ $x^{1 / 2+\varepsilon},-5 / 9 \leq \Re u \leq \varepsilon^{2},|\Im u| \leq 1$. If $x \leq z \leq x+y$, then $z^{u}=x^{u}+$ $O\left(y x^{\varepsilon^{2}-1}\right)$, hence from Proposition $\mathrm{C}$ we get

$$
\sum_{x<n \leq x+y} \gamma^{u}(n)=y H(u) x^{u}+O\left(y x^{-\varepsilon / 10}+x^{1 / 5+2 \varepsilon / 3}\right) .
$$

Taking the $k$ th derivative of both sides of (3.8) and then letting $u=0$ we get

$$
\sum_{x<n \leq x+y} \log ^{k} \gamma(n)=y \sum_{j=0}^{k}\left(\begin{array}{l}
k \\
j
\end{array}\right) H^{(j)}(0) \log ^{k-j} x+O\left(y x^{-\varepsilon / 10}+x^{1 / 5+2 \varepsilon / 3}\right) .
$$

Note that $\log n=\log x+O\left(x^{-1} y\right)$ for any $x<n \leq x+y$. Thus we have

$$
\begin{aligned}
\sum_{x<n \leq x+y} \lambda^{-k}(n) & =y \sum_{j=0}^{k}\left(\begin{array}{l}
k \\
j
\end{array}\right) H^{(j)}(0) \log ^{-j} x+O\left(y x^{-\varepsilon / 10}+x^{1 / 5+2 \varepsilon / 3}\right) \\
& =\sum_{j=0}^{k}\left(\begin{array}{l}
k \\
j
\end{array}\right) H^{(j)}(0) \int_{x}^{x+y} \log ^{-j} z d z+O\left(y x^{-\varepsilon / 10}+x^{1 / 5+2 \varepsilon / 3}\right) .
\end{aligned}
$$

This completes the proof of Theorem 4 .

\section{References}

[1] J. M. De Koninck et N. Doyon, À propos de l'indice de composition des nombres, Monatsh. Math. 139 (2003), 151-167.

[2] J. M. De Koninck and I. Kátai, On the mean value of the index of composition of an integer, ibid. 145 (2005), 131-144.

[3] M. Filaseta and O. Trifonov, The distribution of fractional parts with applications to gap results in number theory, Proc. London Math. Soc. 73 (1996), 241-278.

[4] A. Ivić, The Riemann Zeta-Function, Wiley, 1985.

[5] W. G. Zhai, Short interval results for a class of integers, Monatsh. Math. 140 (2003), 233-257.

School of Mathematical Sciences

Shandong Normal University

Jinan, Shandong, 250014

P.R. China

E-mail: zhaiwg@hotmail.com 